Article

\title{
Effects of Temperature Factors on Resistance against Pine Wood Nematodes in Pinus thunbergii, Based on Multiple Location Sites Nematode Inoculation Tests
}

\author{
Taiichi Iki ${ }^{1, *,+}$, Koji Matsunaga $^{2, *,+}{ }^{\mathbb{D}}$, Tomonori Hirao ${ }^{3}$, Mineko Ohira ${ }^{3}$, Taro Yamanobe ${ }^{3}$, \\ Masakazu G Iwaizumi ${ }^{4}$, Masahiro Miura ${ }^{4}$, Keiya Isoda ${ }^{3}$, Manabu Kurita ${ }^{2}$, Makoto Takahashi ${ }^{3}$ \\ and Atsushi Watanabe ${ }^{5}$ \\ 1 Tohoku Regional Breeding Office, Forest Tree Breeding Center, Forestry and Forest Products Research \\ Institute, 95 Osaki, Takizawa, Iwate 020-0621, Japan \\ 2 Kyushu Regional Breeding Office, Forest Tree Breeding Center, Forestry and Forest Products Research \\ Institute, 2320-5 Suya, Koshi, Kumamoto 861-1102, Japan; mkuri@affrc.go.jp \\ 3 Forest Tree Breeding Center, Forestry and Forest Products Research Institute, 3809-1 Ishii, Juo, Hitachi, \\ Ibaraki 319-1301, Japan; hiratomo@affrc.go.jp (T.H.); sasamine@affrc.go.jp (M.O.); \\ yamanobe@affrc.go.jp (T.Y.); keiso@affrc.go.jp (K.I.); makotot@affrc.go.jp (M.T.) \\ 4 Kansai Regional Breeding Office, Forest Tree Breeding Center, Forestry and Forest Products Research \\ Institute, 1043, Uetsukinaka, Shoo, Katsuta, Okayama 709-4335, Japan; ganchan@affrc.go.jp (M.G.I.); \\ miumasa@affrc.go.jp (M.M.) \\ 5 Department of Forest Environmental Science, Faculty of Agriculture, Kyushu University, 744 Motooka, \\ Nishiku, Fukuoka 819-0395, Japan; nabeatsu@agr.kyushu-u.ac.jp \\ * Correspondence: iki@affrc.go.jp (T.I.); makoji@affrc.go.jp (K.M.); \\ Tel.: +81-19-688-4517 (T.I.); +81-96-242-3151 (K.M.) \\ + These authors contributed equally to this work.
}

Received: 22 July 2020; Accepted: 20 August 2020; Published: 24 August 2020

\begin{abstract}
Pine wilt disease (PWD) caused by the pinewood nematode (PWN) (Bursaphelenchus xylophilus (Steiner and Buhrer) Nickle) is a worldwide issue. Infection is considered to be promoted mainly by the increased air temperature, but it is important to investigate whether the effect of high temperature similarly influences the different ranks of resistant clone. In the present study, we conducted PWN inoculation tests using six common open-pollinated families of resistant Pinus thunbergii Parl. The tests were conducted at nurseries of five test sites across Japanese archipelago between 2015 and 2017. Our analysis focused specifically on temperature. Firstly, we examined the effects of test sites, inoculation year, and their interaction on unaffected seedling rate and found that the unaffected seedling rate of all tested pine families decreased as the cumulative temperature increased. We found that the unaffected seedling rate decreased as the cumulative temperature increased for all tested pine families. In general, higher cumulative temperatures were required for having an effect on the unaffected seedling rates of higher PWN-resistant families. Typically, early cumulative temperatures, i.e., 19 days after inoculation, had the greatest effect on the unaffected seedling rates of PWN-resistant pines. However, the relationship between cumulative temperature and predicted unaffected seedling rate follow similar rate for all families. Thus, the order of resistance level is maintained in terms of the cumulative temperature required for having an effect.
\end{abstract}

Keywords: pine wood disease; resistance to pine wood nematode; inoculation test; multisite; cumulative temperature; Pinus thunbergii 


\section{Introduction}

Pine wilt disease is an epidemic disease caused by the invasive pinewood nematode, Bursaphelenchus xylophilus (Steiner and Buhrer) Nickle [1], and vectored by pine sawyer beetles, Monochamus alternatus Hope [2]. PWD is currently a worldwide issue [3], and future climate change could lead to further spread of the disease given that its development depends on temperature and drought [4]. Pinus thunbergii Parl. and Pinus densiflora Sieb. et Zucc., two major planted pine species in Japan, are susceptible to PWN [5]. Thus, Japanese pine forests have been seriously damaged by PWD. The first documented observation of PWD was in Nagasaki Prefecture in 1905 [6]. The disease has subsequently spread to every prefecture in Japan, apart from Hokkaido [7]. As the disease causes widespread damage, Japan began a tree breeding project in 1978 in order to select resistant pine varieties as a countermeasure against PWD. In the first breeding project, conducted from 1978 to 1984 in southwestern Japan, 16 and 92 resistant clones of $P$. thunbergii and P. densiflora were selected, respectively [8-10]. Resistance against PWN was conferred by artificial inoculation, wherein higher survival rates represented greater resistance [9]. Up to March 2019, several related projects have led to the selection of 211 and 288 resistant clones of $P$. thunbergii and $P$. densiflora, respectively [11].

The development of PWD symptoms and mortality after inoculation are affected by the climatic factors, including air temperature [12-14], precipitation [9,15] and light conditions [16]. In particular, temperature is strongly related to PWD, as PWD only occurs in areas where the average temperature exceeds $20^{\circ} \mathrm{C}$ for several weeks [17]. In non-resistant $P$. thunbergii seedlings grown in phytotrons and temperature-controlled greenhouses, PWD symptoms develop faster and mortality rates increase as temperatures rise $[13,14]$. It appears that the effect of temperature on the PWN propagation rate affects the development of PWD symptoms, leading to tree death [13,14]. In nursery-based inoculation tests using open-pollinated families of resistant $P$. thunbergii, survival rates varied substantially among inoculation years and families, suggesting that differences in climate variation at the period of inoculation can influence tree mortality [18-20]. Variation in resistance exist among resistant P. thunbergii clones [9]; however, no studies have yet been conducted to investigate whether the effect of high temperature similarly influences the different ranks of resistant clones. It is important to understand how temperature affects the resistance, especially given the potential effects of future climate change.

To clarify how temperature factors affect the different ranks of resistance against $\mathrm{PWN}$, inoculation tests were conducted over multiple years, from 2015 to 2017, at the nurseries of five test sites in regions with different climates. In addition, we used common open-pollinated families of six resistant $P$. thunbergii clones with different levels of resistance to PWN.

\section{Materials and Methods}

\subsection{Test Sites}

Inoculation tests were conducted at the nurseries of five test sites: Forest Tree Breeding Center (FTBC) Head Quarters in Hitachi, Ibaraki $\left(36.69^{\circ} \mathrm{N}, 140.69^{\circ} \mathrm{E}\right)$; Tohoku Regional Breeding Office, FTBC (TBO) in Takizawa, Iwate $\left(39.83^{\circ} \mathrm{N}, 141.14^{\circ} \mathrm{E}\right)$; Kansai Regional Breeding Office, FTBC (KABO) in Shou-cho, Okayama (35.06 $\left.{ }^{\circ} \mathrm{N}, 134.11^{\circ} \mathrm{E}\right)$; Shikoku Breeding Stock Garden, KABO, FTBC (SSG) in Kami, Kochi ( $\left.33.61^{\circ} \mathrm{N}, 133.70^{\circ} \mathrm{E}\right)$; and Kyushu Regional Breeding Office, FTBC (KYBO) in Koshi, Kumamoto $\left(32.88^{\circ} \mathrm{N}, 130.74^{\circ} \mathrm{E}\right)$. Their locations are shown in Figure 1. The mean temperatures for the current inoculation test periods at each test site are shown in Figure S1. 


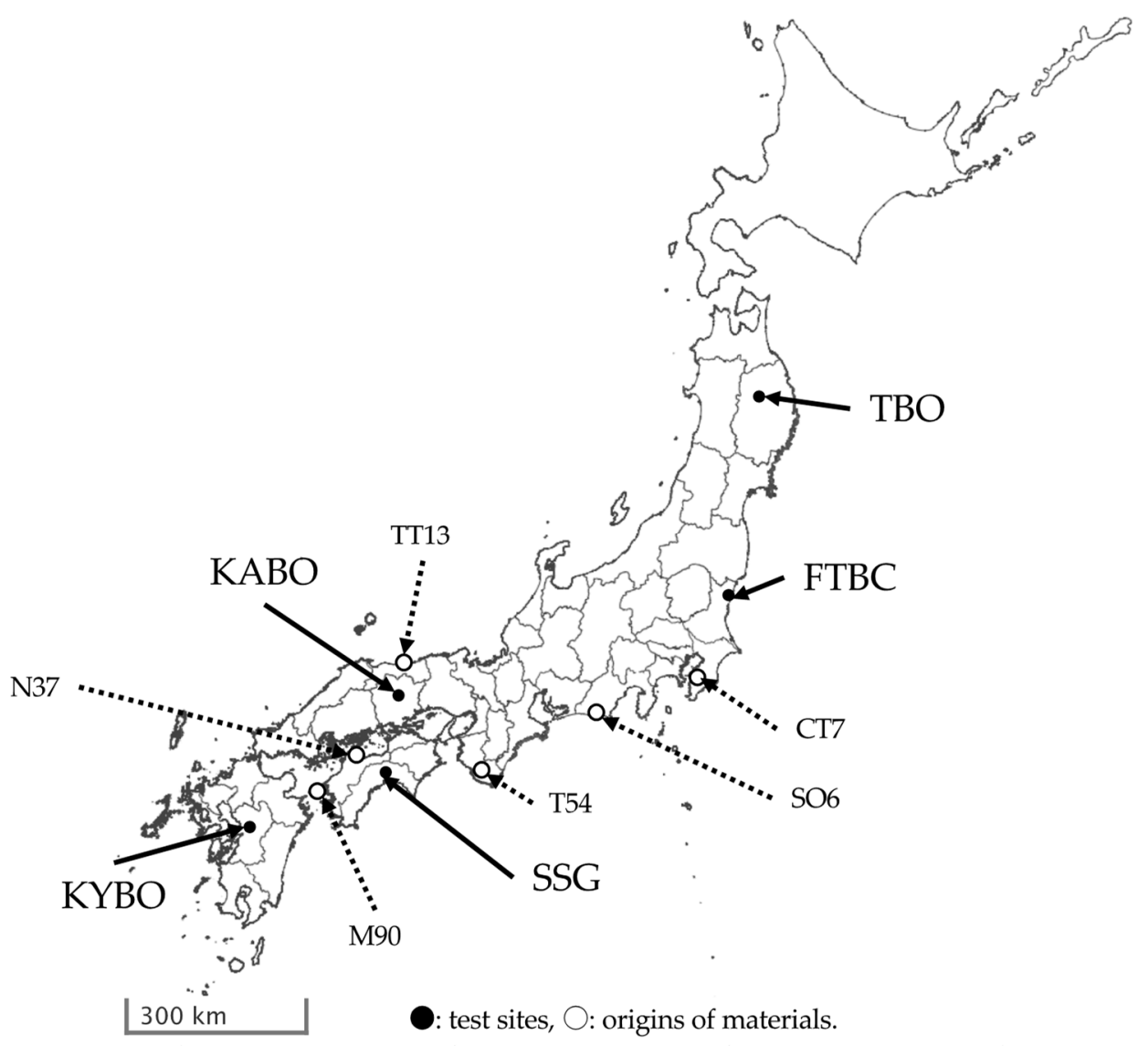

Figure 1. Location of each test site and origin of materials used in the present study. This map was created from the blank map published by Geospatial Information Authority of Japan (https: //www.gsi.go.jp/tizu-kutyu.html). Test site abbreviations: Tohoku Regional Breeding Office (TBO), Forest Tree Breeding Center (FTBC), Kansai Regional Breeding Office (KABO), Shikoku Breeding Stock Garden (SSG), and Kyushu Regional Breeding Office (KYBO). Family name abbreviations: Misaki 90 (M90), Namikata 37 (N37), Tanabe 54 (T54), Shizuoka (Oosuka) 6 (SO6), Chiba (Tomiura) 7 (CT7), and Tottori (Tottori) 13 (TT13).

\subsection{Plant Material and PWN Inoculation}

Open-pollinated families of six resistant $P$. thunbergii clones were used in the present study (Table 1), and the same seed-lots were used for all test sites across the three-year inoculations. The six families used in this study were Misaki 90 (M90), Namikata 37 (N37), Tanabe 54 (T54), Shizuoka (Oosuka) 6 (SO6), Chiba (Tomiura) 7 (CT7), and Tottori (Tottori) 13 (TT13). The origins of these families are shown in Figure 1. Of these, three clones (M90, N37, and T54) were selected in the first breeding project and had already been evaluated for their resistance rank [9]. The other clones (SO6, CT7, and TT13) were recently selected from other regions, and their resistance against PWN had not yet been evaluated.

Seedlings were grown using the same procedure until the inoculation test, except for at TBO. The standard method was as follows: seeds were sown on the seedbed in spring one year before the inoculation test; the seedlings were then transplanted to a nursery in the following spring; then the inoculation test was conducted with two replications. As the TBO location is colder than the other test sites, the growth of seedlings was slower; therefore, to promote the growth of seedlings and allow them to be inoculated at the 2-year-old stage, the seeds were sown individually in a nursery with two replications, and the seedlings were not transplanted for about 16 months, until the inoculation test was conducted. 
Table 1. Resistant $P$. thunbergii families used in this study.

\begin{tabular}{|c|c|c|c|c|c|c|c|c|c|c|c|c|c|c|c|}
\hline \multirow{3}{*}{$\begin{array}{c}\text { Family } \\
\text { Name }\end{array}$} & \multirow{3}{*}{$\begin{array}{l}\text { Resistance } \\
\text { Ranking * }\end{array}$} & \multicolumn{14}{|c|}{ Number of Seedlings Used in the Inoculation Tests at Each Study Site in Each Year } \\
\hline & & \multicolumn{5}{|c|}{2015} & \multicolumn{5}{|c|}{2016} & \multicolumn{4}{|c|}{2017} \\
\hline & & ТВО & FTBC & КАВО & SSG & KYBO & ТВО & FTBC & КАВО & SSG & KYBO & ТВО & FTBC & КАВO & KYBO \\
\hline M90 & 4 & 27 & 37 & 22 & 31 & 32 & 35 & 40 & 20 & 40 & 22 & 33 & 34 & 25 & 24 \\
\hline N37 & 4 & 28 & 32 & 23 & 29 & 32 & 27 & 32 & 28 & 35 & 16 & 30 & 29 & 23 & 19 \\
\hline T54 & 2 & 44 & 40 & 33 & 40 & 39 & 57 & 39 & 41 & 37 & 37 & 55 & 40 & 21 & 36 \\
\hline SO6 & - & 37 & 40 & 28 & 40 & 32 & 50 & 40 & 37 & 40 & 34 & 44 & 37 & 28 & 17 \\
\hline CT7 & - & 37 & 37 & 32 & 40 & 40 & 35 & 40 & 7 & 40 & 27 & 35 & 39 & 32 & 29 \\
\hline TT13 & - & 40 & 40 & 27 & 19 & 39 & 50 & 39 & 11 & 40 & 32 & 28 & 39 & 31 & 26 \\
\hline
\end{tabular}

* Resistance ranking was evaluated by using a least squares means method based on the survival rates following inoculation tests on open-pollinated families. -: Resistance not evaluated. Test site abbreviations: Tohoku Regional Breeding Office (TBO), Forest Tree Breeding Center (FTBC), Kansai Regional Breeding Office (KABO), Shikoku Breeding Stock Garden (SSG), Kyushu Regional Breeding Office (KYBO). Family name abbreviations: Misaki 90 (M90), Namikata 37 (N37), Tanabe 54 (T54), Shizuoka (Oosuka) 6 (SO6), Chiba (Tomiura) 7 (CT7), and Tottori (Tottori) 13 (TT13).

The number of seedlings at each test site is shown in Table 1. At SSG, the growth of seedlings was poor due to insect damage (unrelated to PWN) in 2017; hence, we could not conduct inoculation tests at SSG in 2017. In general, the number of seedlings in each of the families ranged from 7 to 57. It has been reported that the size of $P$. thunbergii seedlings affects their post-inoculation survival $[20,21]$; therefore, we also measured seedling height immediately before inoculation testing.

The virulent isolate of the PWN, Ka4 [22], which has been widely used in the resistance breeding program in Japan, was used in the present study. A $50-\mu \mathrm{L}$ suspension containing 5000 PWNs (a density of 100,000 PWN/mL) was inoculated onto wounds in the basal axes of subjects, which were made by peeling the cortex with a knife and scratching the xylem with a fine saw. At each test site, inoculation was conducted in early July in each of the three years from 2015 to 2017 (Table S1). The seedling age at the time of inoculation was approximately 15-16 months (based on time after sowing).

\subsection{Symptom Observation}

Symptoms were observed approximately ten weeks after inoculation (Table S1) and classified by visual judgement for each inoculated seedling class as follows: 0: no symptom, 1: browning of needles on one or more branches, 2: browning of all needles. From the results, the unaffected seedling rate was calculated using the following equation:

Unaffected seedling rate $(\%)=$ number of seedlings in symptom class $0 /$ number inoculated seedlings $\times 100$

\subsection{Temperature Data}

The temperature data used in the present study were collected from the Automated Meteorological Data Acquisition System (AMeDAS) provided by the Japanese Meteorological Agency [23]. We collected climate data from the AMeDAS station nearest to each of test site; Morioka $\left(39.70^{\circ} \mathrm{N}, 141.16^{\circ} \mathrm{E}\right)$ for TBO, Hitachi $\left(36.58^{\circ} \mathrm{N}, 140.65^{\circ} \mathrm{E}\right)$ for FTBC, Tsuyama $\left(35.07^{\circ} \mathrm{N}, 134.02^{\circ} \mathrm{E}\right)$ for KABO, Kochi $\left(33.57^{\circ} \mathrm{N}\right.$, $\left.133.55^{\circ} \mathrm{E}\right)$ for SSG and Kumamoto $\left(32.82^{\circ} \mathrm{N}, 130.70^{\circ} \mathrm{E}\right)$ for $\mathrm{KYBO}$.

Hourly temperature data were collected from each station. Temperature data for analysis were labelled CT, CT20, CT25, and CT30, which represent cumulative temperature with different thresholds $\left(0{ }^{\circ} \mathrm{C}, 20^{\circ} \mathrm{C}, 25^{\circ} \mathrm{C}\right.$, and $\left.30^{\circ} \mathrm{C}\right)$ from the day of inoculation to the $n$ day after inoculation (DAI). They were calculated using the following the formulae:

$$
\text { CT at } n \text { DAI }=\sum_{j=1}^{n} \sum_{i=1}^{24}\left(T H_{i j} \times \delta_{i j}\right) / n \quad \delta_{i j}=\left\{\begin{array}{l}
1,\left(T H_{i j}>0\right) \\
0,\left(T H_{i j} \leq 0\right)
\end{array}\right.
$$




$$
\begin{array}{ll}
\text { CT2 at } n \text { DAI }=\sum_{j=1}^{n} \sum_{i=1}^{24}\left(\left(T H_{i j}-20\right) \times \delta_{i j}\right) / n & \delta_{i j}=\left\{\begin{array}{l}
1,\left(\left(T H_{i j}-20\right)>0\right) \\
0,\left(\left(T H_{i j}-20\right) \leq 0\right)
\end{array}\right. \\
\text { CT25 at } n \text { DAI }=\sum_{j=1}^{n} \sum_{i=1}^{24}\left(\left(T H_{i j}-25\right) \times \delta_{i j}\right) / n & \delta_{i j}=\left\{\begin{array}{l}
1,\left(\left(T H_{i j}-25\right)>0\right) \\
0,\left(\left(T H_{i j}-25\right) \leq 0\right)
\end{array}\right. \\
\text { CT30 at } n \text { DAI }=\sum_{j=1}^{n} \sum_{i=1}^{24}\left(\left(T H_{i j}-30\right) \times \delta_{i j}\right) / n & \delta_{i j}=\left\{\begin{array}{l}
1,\left(\left(T H_{i j}-30\right)>0\right) \\
0,\left(\left(T H_{i j}-30\right) \leq 0\right)
\end{array}\right.
\end{array}
$$

The inoculation day was defined as Day 0 , and the climate data from this day were not used in our analysis. Temperature data were therefore analyzed from the 1 DAI to the 35 DAI.

\subsection{Statistical Analysis}

All statistical analyses were conducted in R version 3.6.1 [24]. To examine the effects of test site and inoculation year on the unaffected seedling rate of $P$. thunbergii families, logistic regression analysis was conducted using a generalized mixed linear model via the "glmer" function of the lme4 package [25]. Appropriate models were selected using Akaike information criterion (AIC) values obtained with the "dredge" function of the MuMIn package [26]. In this analysis, test site, inoculation year, seedling height (mean value in replication), and the interaction between test site and inoculation year were fixed effects; family, replication, the interaction between family and test site, and the interaction between family and inoculation year were the random effects. Since the unaffected seedling rate showed a binomial distribution, the family was set as "binomial" using the logit link function in R. To identify significant differences in the fixed effects, the "ANOVA" function in the car package [27] was used to conduct deviance analysis (Type II test, level of significance was $p<0.01$ ) on the test site, inoculation year, and interaction between the test site and inoculation year. In addition, for test site and inoculation year, multiple comparison analysis was performed using the Tukey method (level of significance was $p<0.05$ ) via the "glht" and "cld" functions in the multcomp package [28]. Furthermore, the variance component of each random effect was calculated using the "VarCorr" function in the lme4 package. The best linear unbiased prediction (BLUP) value for each family was calculated using the "ranef" function in the lme4 package for unaffected seedling rate.

The correlation coefficients between each temperature factor and the unaffected seedling rate of each family were calculated using product-moment correlation via the "cor.test" function. The mean of correlation coefficients for all families was calculated in each temperature factor.

To analyze the post-inoculation temperature factors that affected the unaffected seedling rates, logistic regression analysis was conducted using a generalized mixed linear model via the "glmer" function of the lme4 package. The model was again selected using AIC values obtained with the "dredge" function in the MuMIn package according to a model that excluded the temperature factor (i.e., the "Null" model). In this analysis, the unaffected seedling rate was the response variable; seedling height (mean value in replication) and individual post-inoculation temperature factors were the fixed effects among the explanatory variables; family, test site, inoculation year, repetition, the interaction between family and test site, the interaction between inoculation family and inoculation year, and the interaction between test site and inoculation year were the random effects among the explanatory variables. Family was again set as "binomial" using the logit link function. Following logistic regression analyses, the AIC values of all models were calculated using the "AIC" function. We also used the function "predict", to predict the unaffected seedling rate under the optimal climatic conditions selected by the analysis. At that time, the prediction was performed using the same seedling height (overall mean across all test sites, years, and families), considering the effect of seedling height on unaffected seedling rate. 


\section{Results}

\subsection{Seedling Heights}

Seedling heights (with standard deviations) prior to the inoculation tests are shown in Figure 2. The overall mean of seedling heights was $26.6 \mathrm{~cm}$ across five test sites, three years, and six families. Mean seedling heights for each family were $28.3 \mathrm{~cm}$ for M90, $27.2 \mathrm{~cm}$ for N37, $23.4 \mathrm{~cm}$ for T54, $25.6 \mathrm{~cm}$ for SO6, $27.7 \mathrm{~cm}$ for CT7, and $28.0 \mathrm{~cm}$ for TT13 across five test sites and three years. Seedlings tended to be taller in TBO compared to in the other test sites.
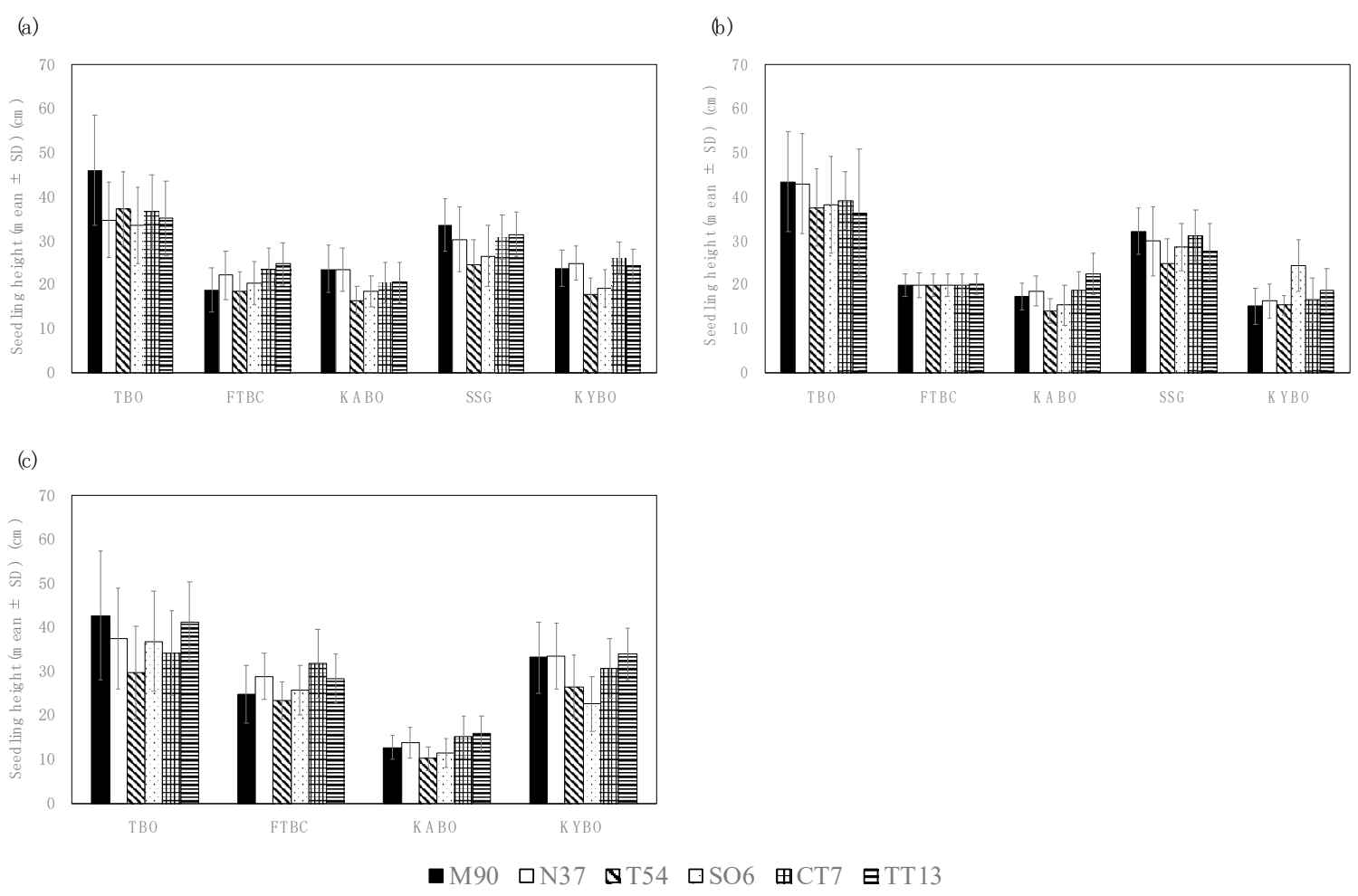

Figure 2. Mean seedling heights of $P$. thunbergii families before inoculation with PWNs in: (a) 2015, (b) 2016, (c) 2017. Error bars represent standard deviations (SD). Test site abbreviations: Tohoku Regional Breeding Office (TBO), Forest Tree Breeding Center (FTBC), Kansai Regional Breeding Office (KABO), Shikoku Breeding Stock Garden (SSG), and Kyushu Regional Breeding Office (KYBO). Family name abbreviations: Misaki 90 (M90), Namikata 37 (N37), Tanabe 54 (T54), Shizuoka (Oosuka) 6 (SO6), Chiba (Tomiura) 7 (CT7), and Tottori (Tottori) 13 (TT13).

\subsection{Unaffected Seedling Rates}

The unaffected seedling rates are shown in Figure 3. The mean of unaffected seedling rates for the six families in 2015 were $72.3 \%$ for TBO, 56.2\% for FTBC, 21.5\% for KABO, 29.0\% for SSG, and 20.5\% for KYBO. In 2016, the means of unaffected seedling rates for all families were $74.3 \%$ for $\mathrm{TBO}, 65.8 \%$ for $\mathrm{FTBC}, 7.4 \%$ for $\mathrm{KABO}, 23.7 \%$ for SSG, and 6.7\% for KYBO. The mean of unaffected seedling rates in 2017 for all families were 39.1\% for TBO, 51.6\% for FTBC, 2.9\% for KABO, and 13.8\% for KYBO. The mean unaffected seedling rates for each family were $52.7 \%$ for M90, 46.9\% for N37, 27.1\% for T54, $24.6 \%$ for SO6, $14.5 \%$ for CT7, and $42.2 \%$ for TT13 across five test sites and three inoculation years. Across the three years, the unaffected seedling rate was higher at TBO and FTBC than at KABO, SSG, and KYBO. 

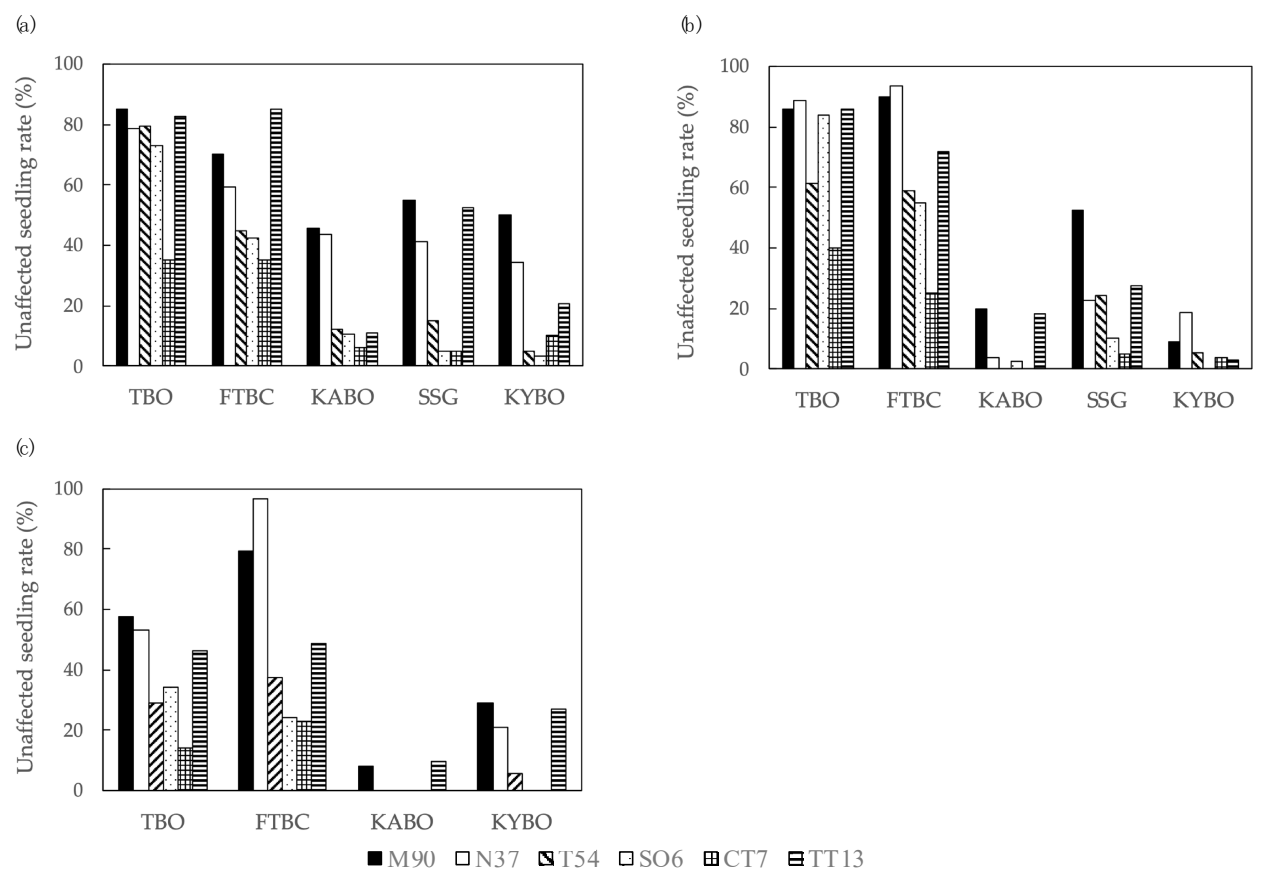

Figure 3. Unaffected seedling rate of $P$. thunbergii families before inoculation with PWNs in: (a) 2015, (b) 2016, (c) 2017. Test site abbreviations: Tohoku Regional Breeding Office (TBO), Forest Tree Breeding Center (FTBC), Kansai Regional Breeding Office (KABO), Shikoku Breeding Stock Garden (SSG), and Kyushu Regional Breeding Office (KYBO). Family name abbreviations: Misaki 90 (M90), Namikata 37 (N37), Tanabe 54 (T54), Shizuoka (Oosuka) 6 (SO6), Chiba (Tomiura) 7 (CT7), and Tottori (Tottori) 13 (TT13).

The deviance and significance of the test site, inoculation year, and interaction between test site and inoculation year are shown in Table 2. Significant differences $(p<0.01)$ in unaffected seedling rates were observed according to test site, inoculation year, and the interaction between test site and inoculation year. Multiple comparison analysis (using the Tukey method) among the test sites showed that the unaffected seedling rates at TBO and FTBC were significantly higher than the rates at KABO, SSG, and KYBO $(p<0.05)$. A similar analysis of inoculation years showed that the unaffected seedling rates in 2015 and 2016 were significantly higher than the rate in $2017(p<0.05)$. From the estimated proportions of the variance components of each random effect, family accounted for $>90 \%$ of all variance components; in contrast, replication and the interaction between family and inoculation year were almost zero. The BLUP values of unaffected seedling rate in each family were as follows: 1.06 for M90, 0.73 for N37, -0.25 for T54, -0.52 for SO6, -1.40 for CT7, and 0.42 for TT13.

Table 2. Effects of site and inoculation year on unaffected seedling rate analyzed using a generalized linear mixed model.

\begin{tabular}{ccccc}
\hline & Deviance & $\begin{array}{c}\text { Degree of } \\
\text { Freedom }\end{array}$ & \multicolumn{2}{c}{$\begin{array}{c}\text { Significance } \\
\operatorname{Pr}\left(>\chi^{2}\right)\end{array}$} \\
\hline Seedling height & 10.369 & 1 & 0.0013 & $p<0.01$ \\
Test site & 242.816 & 4 & $<2.2 \times 10^{-16}$ & $p<0.01$ \\
Year & 101.763 & 2 & $<2.2 \times 10^{-16}$ & $p<0.01$ \\
Test site $\times$ Year & 29.450 & 7 & 0.0001 & $p<0.01$ \\
\hline
\end{tabular}

\subsection{Analysis of Temperature Factors Affecting Unaffected Seedling Rate}

For all temperature factors, all correlation coefficients values were negative values (Figure 4). The correlation coefficients of CT, CT20, and CT25 were higher than those of CT30. The highest 
correlation coefficient in all temperature factors was CT at the 6 DAI $(r=-0.804)$. For CT20, the highest correlation coefficient was observed on the 13 DAI $(r=-0.786)$. For CT25, the highest correlation coefficient was observed on the 14 DAI ( $r=-0.746)$. In CT, CT20, and CT25, the correlation coefficients tended to gradually decrease after showing the highest value.

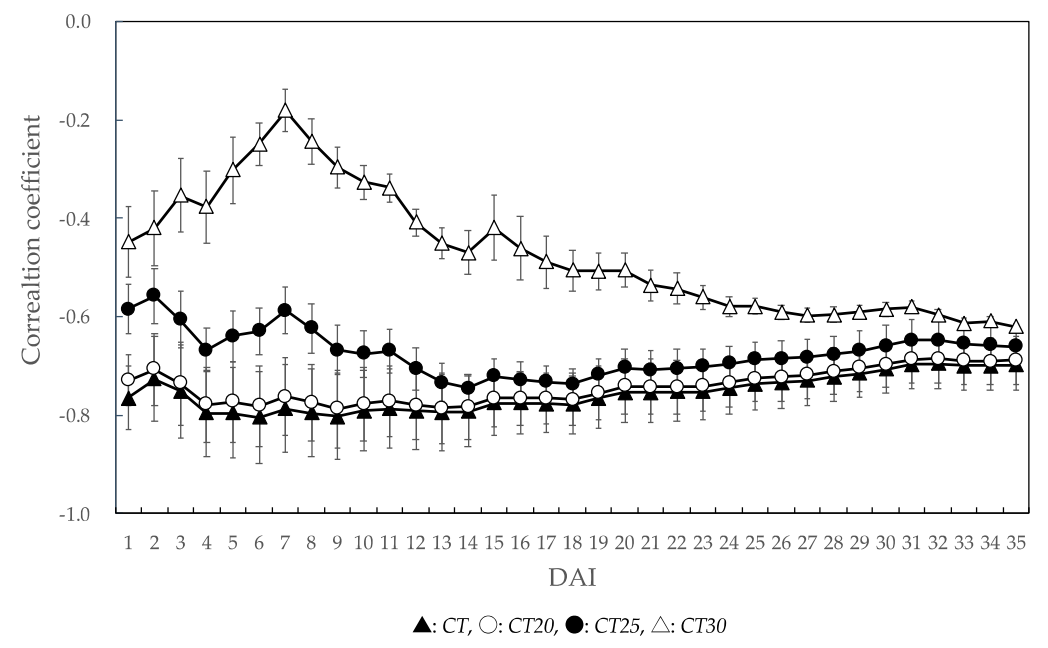

Figure 4. Changes in the correlation coefficients for each temperature factor according to the unaffected seedling rate of each $P$. thunbergii family. The value of the correlation coefficient in this figure is the mean value of six families. Error bars represent SD. DAI: days after inoculation. Temperature factor abbreviations: $C T, C T 20, C T 25$, and CT30 are represented cumulative temperature with different thresholds $\left(0{ }^{\circ} \mathrm{C}, 20^{\circ} \mathrm{C}, 25^{\circ} \mathrm{C}\right.$, and $\left.30^{\circ} \mathrm{C}\right)$ from the day of inoculation to the $n$ DAI.

In the analysis of the effects of post-inoculation temperature factors on the unaffected seedling rate of $P$. thunbergii ten weeks after inoculation (Figure 5), the AICs of CT, CT20, and CT25 were lower than that of CT30. The lowest AIC for all temperature factors for CT25 was on the 19 DAI (AIC =665). For CT20, the lowest AIC was observed on the 19 DAI $(\mathrm{AIC}=666)$. The correlation coefficients 19 DAI were -0.766 for $C T,-0.756$ for $C T 20$, and -0.719 for CT25. In all temperature factors, the AICs tended to gradually increase after reaching the lowest value.

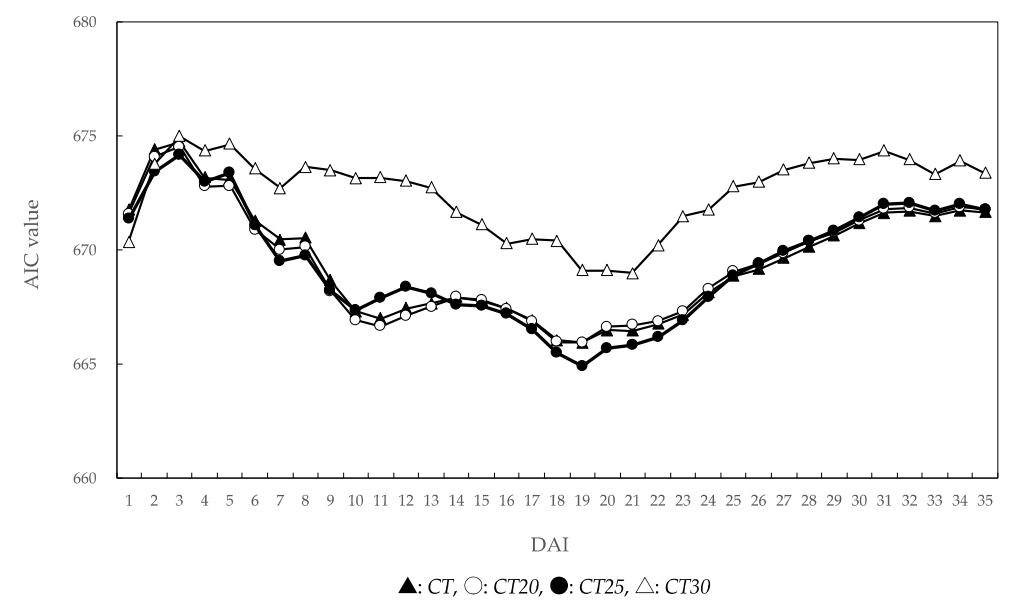

Figure 5. Changes in Akaike information criterion (AIC) values calculated from a generalized linear mixed model including temperature. DAI: days after inoculation. Temperature factor abbreviations: CT, CT20, CT25, and CT30 represented cumulative temperature with different thresholds $\left(0{ }^{\circ} \mathrm{C}, 20^{\circ} \mathrm{C}\right.$, $25^{\circ} \mathrm{C}$, and $30^{\circ} \mathrm{C}$ ) from the day of inoculation to the $n$ DAI. 
When the family-level unaffected seedling rate was predicted by the model including CT25 on the 19 DAI, negative relationships were shown in all families (Figure 6). The observed correlation coefficients were -0.760 for M90, -0.774 for N37, -0.792 for T54, -0.800 for SO6, -0.767 for CT7, and -0.789 for TT13. Unaffected seedling rates for each family apparently decrease as the cumulative temperature rises. At all ranges of cumulative temperature, the unaffected seedling rate was predicted to be higher in the order of M90, N37, TT13, T54, SO6, and CT7. This order of families matched that of the BLUP results.

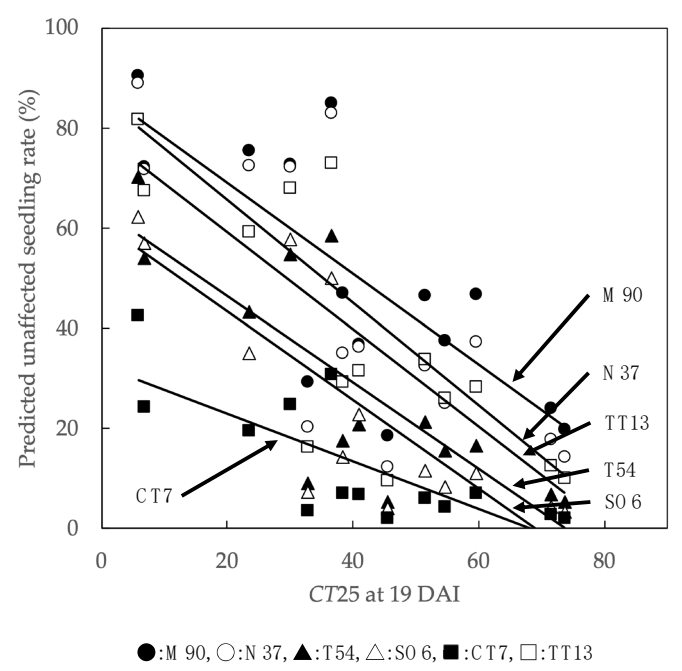

Figure 6. Relationship between optimum temperature factor and predicted unaffected seedling rate. The predicted unaffected seedling calculated from a generalized linear mixed model including optimum temperature factor (CT25 at 19 DAI). DAI: days after inoculation. Family name abbreviations: Misaki 90 (M90), Namikata 37 (N37), Tanabe 54 (T54), Shizuoka (Oosuka) 6 (SO6), Chiba (Tomiura) 7 (CT7), and Tottori (Tottori) 13 (TT13).

\section{Discussion}

\subsection{Differences in PWN-Resistance among Test Sites and Inoculation Years}

In inoculation tests conducted across multiple nurseries, it has been suggested that the unaffected seedling rates of $P$. thunbergii resistant families vary greatly depending on climatic factors related to the test site and the inoculation year [18-20]. Our results support this suggestion: the unaffected seedling rates differed among test sites and inoculation years, thus the PWN resistance of $P$. thunbergii was apparently affected by climatic factors at the test site and during the inoculation year. However, the variance component of resistant families was substantially larger for the pine family than for the interaction between the family and the test site or the interaction between the family and the inoculation year, thus the rank of the unaffected seedling rate of six families was stable even though the climatic conditions were different in the three years studied. The ranking of unaffected seedling rate was in the following order: M90, N37, TT13, T54, SO6, and CT7. The highest levels of resistance to PWN also apparently followed this order. The variation in unaffected seedling rate in the present study was large, ranging from $2.9 \%$ to $74.3 \%$. This wide range may have been created by the differing temperature factors at the five sites over three years.

\subsection{Temperature Factors Affecting PWN-Resistance}

Previous studies have shown that PWN migration and propagation in P.thunbergii trees are important for symptom development of PWD and for mortality [12-14]. It has also been shown that cumulative temperature, especially at $25-30{ }^{\circ} \mathrm{C}$, is important for the propagation of inoculated PWNs in pine trees $[13,14]$. In the present study, the AIC value was lowest in CT25 on the $19 \mathrm{DAI}$, and the 
correlation coefficient also showed a high value. Our results suggest that a cumulative temperature of $25{ }^{\circ} \mathrm{C}$ or higher affects the unaffected seedling rate of resistant $P$. thunbergii after inoculation, which is the same temperature range with propagation of PWN after inoculation $[13,14]$.

Not only CT25 at 19 DAI but also CT and CT20 at 19 DAI showed low AICs. From this result, the temperature factor, the cumulative temperature until 19 DAI, seems to be considerably related to the unaffected seedling rate. Hirao et al. [29] investigated gene expression after inoculation using grafted clones of PWN-resistant and PWN-susceptible P. thunbergii and reported that genes related to cell wall strength were significantly higher in resistant $P$. thunbergii clones at 14 DAI than in susceptible pine clones at 7 DAI. Kusumoto et al. [30] also investigated histological response, tissue damage expansion, and PWN distribution after inoculation using grafted clones of PWN-resistant and PWN-susceptible $P$. thunbergii, and reported that PWN propagation was suppressed immediately after inoculation, and that proteins related to cell wall strength were highly expressed in resistant pine clones relative to susceptible pine clones. According to our findings, in general, cumulative temperature has a greater effect on seedling rate soon after inoculation, e.g., 19 DAI. Therefore, in resistant P. thunbergii, we suggest that if the cumulative temperature is low soon after inoculation, the propagation of PWNs in the $P$. thunbergii tree is likely to be insufficient to cause death in the infested trees due to the protective reaction.

As the correlation coefficients between the predicted unaffected seedling rate and the CT25 at 19 DAI were negative for all families, unaffected seedling rate apparently decreases as the cumulative temperature $\left(25^{\circ} \mathrm{C}\right.$ or higher) rises. However, the cumulative temperature of $25{ }^{\circ} \mathrm{C}$ or higher that affected the unaffected seedling rate differed among resistant pine families. The more resistant the family was, the higher the cumulative temperature required to effect the unaffected seedling rate. We suggest that these family differences in the cumulative temperature that affect the unaffected seedling rate are related to the propagation of PWNs in the trees after inoculation. Although the resistance mechanism to PWNs in $P$. thunbergii has yet to be confirmed, it has been suggested that post-inoculation migration and propagation of PWNs is restricted in resistant pine trees $[21,31,32]$. Among the families studied here, for example, M90 is highly resistant to PWN, so they perhaps restricted the propagation of PWN after inoculation more than other families. Therefore, higher cumulative temperatures were required for sufficient propagation for the disease develop.

\subsection{Application to the Resistance Breeding Program}

The results of the present study suggest that the unaffected seedling rates of PWN-resistant P. thunbergii families decrease as the cumulative temperature $\left(25^{\circ} \mathrm{C}\right.$ or higher $)$ increases. In addition, the effects of cumulative temperature appear to occur soon after PWN inoculation, i.e., at 19 DAI. However, these effects differed in each of the families tested here. That is, the higher the resistance to PWN, the higher the cumulative temperature needed.

In Japan, resistant clones selected from the field (first-generation) are crossed, and secondgeneration resistant $P$. thunbergii are then selected. Candidate trees from the second generation of resistant clones are estimated to have higher levels of resistance than those of the first generation $[33,34]$. Given our results, it will be necessary to increase the selection criteria in future, when selecting the second generation, in order to effectively evaluate the resistance level. For example, we suggest that inoculation tests should be conducted when high temperatures are expected.

\section{Conclusions}

We conducted PWN inoculation tests on six common open-pollinated families of resistant P. thunbergii in the nurseries of five test sites from 2015 to 2017, in order to consider the effects of temperature factors on PWN resistance. The results suggested that the unaffected seedling rates of PWN-resistant $P$. thunbergii families decrease as the cumulative temperature increases. However, the cumulative temperatures that affected unaffected seedling rate differed among families. Namely, higher cumulative temperatures were required for the effect in more highly PWN-resistant pines. 
In addition, early cumulative temperatures had greater effects on the unaffected seedling rate of PWN-resistant $P$. thunbergii.

Supplementary Materials: The following are available online at http://www.mdpi.com/1999-4907/11/9/922/s1, Figure S1: Mean temperatures for the current inoculation test period at each test site in 2015, 2016, and 2017. This Figure was created from data obtained from AMeDAS (Automated Meteorological Data Acquisition System) of Japan Meteorological Agency (http://www.data.jma.go.jp/obd/stats/etrn/index.php). Test site abbreviations: Tohoku Regional Breeding Office (TBO), Forest Tree Breeding Center (FTBC), Kansai Regional Breeding Office (KABO), Shikoku Breeding Stock Garden (SSG), and Kyushu Regional Breeding Office (KYBO). Table S1: Dates of Inoculation and investigations.

Author Contributions: Conceptualization, all authors; material management and investigation, T.I., K.M., T.Y., M.O., M.G.I., M.M., K.I., M.K.; data curation and writing—original draft preparation, T.I. and K.M.; writing-review and editing, K.M., T.H., T.Y., M.O., M.G.I., M.M., M.T. and A.W.; project administration, T.H., M.T. and A.W. All authors have read and agreed to the published version of the manuscript.

Funding: The present study is part of the project on 'Project to advance the development of technology for varieties of Japanese black pine and red pine resistant to the pine wood nematode' supported by Forestry Agency, Ministry of Agriculture, Forestry and Fisheries, Japan.

Acknowledgments: We thank Hiroshi Hoshi (FTBC, FFPRI) for his well coordination of the research project, Jin'ya Nasu (TBO, FTBC, FFPRI) and Michinari Matsushita (FTBC, FFPRI) for advice of statistical analysis. We thank our colleagues in the field management section of TBO, FTBC, KABO, SSG and KYBO, in FTBC, FFPRI for management of materials in each nursery.

Conflicts of Interest: The authors declare no conflict of interest.

\section{References}

1. Kiyohara, T.; Tokushige, Y. Inoculation experiments of a nematode, Bursaphelenchus sp., onto pine trees. J. Jpn. For. Soc. 1971, 5, 210-218, (In Japanese with English summary).

2. Mamiya, Y.; Endo, N. Transmission of Bursaphelenchus lignicolus (Nematoda: Aphelenchoididae) by Monochamus alternatus (Coleoptera: Cerambycidae). Nematologica 1972, 18, 159-162. [CrossRef]

3. Zhao, B.G.; Futai, K.; Sutherland, J.R.; Takeuchi, Y. Pine Wilt Disease; Springer: New York, NY, USA, 2008.

4. Hirata, A.; Nakamura, K.; Nakao, K.; Kominami, Y.; Tanaka, N.; Ohashi, H.; Takano, K.T.; Takeuchi, W.; Matsui, T. Potential distribution of pine wilt disease under future climate change scenarios. PLoS ONE 2017, 12, e0182837. [CrossRef] [PubMed]

5. Futai, K.; Furuno, T. The variety of resistance among pine-species to pine wood nematode, Bursaphelenchus lignicolus. Bull. Kyoto Univ. For. 1979, 51, 23-36, (In Japanese with English summary).

6. Yano, M. Investigation on the causes of pine mortality in Nagasaki Prefecture. Sanrinkoho 1913, 4, 1-14. (In Japanese)

7. Nakamura, K.; Otsuka, I. Forest Pest Management Leading to Establishment of Forestry Business: Pine Wilt Disease Unites the Locality; Japan Forest Investigation Company: Tokyo, Japan, 2019. (In Japanese)

8. Fujimoto, Y.; Toda, T.; Nishimura, K.; Yamate, H.; Fuyuno, S. Breeding project on resistance to the pine Wood nematode-An outline of the research and the achievement of the project for ten years. Bull. For. Tree. Breed. Center. 1989, 7, 1-84, (In Japanese with English summary).

9. Toda, T. Studies on the breeding for resistance to the pine wilt disease in Pinus densiflora and P. thunbergii. Bull. For. Tree. Breed. Inst. 2004, 20, 83-217, (In Japanese with English summary).

10. Kurinobu, S. Current status of resistance breeding of Japanese pine species to pine wilt disease. For. Sci. Tec. 2008, 4, 51-57. [CrossRef]

11. Forest Tree Breeding Center. Annual Report; Forest Tree Breeding Center: Hitachi, Japan, 2020. (In Japanese)

12. Kiyohara, T. Effect of temperature on the disease incidence of pine seedlings inoculated with Bursaphelenchus lignicolus. Trans. Ann. Meet. Jpn. For. Soc. 1973, 84, 334-335, (In Japanese with English summary).

13. Ichihara, Y.; Fukuda, K.; Suzuki, K. Early symptom development and histological changes associated with migration of Bursaphelenchus xylophilus in seedling tissues of Pinus thunbergii. Plant Dis. 2000, 84, 675-680. [CrossRef]

14. Yamaguchi, R.; Matsunaga, K.; Watanabe, A. Influence of temperature on pine wilt disease progression in Pinus thunbergii seedlings. Eur. J. Plant. Pathol. 2020, 156, 581-590. [CrossRef] 
15. Suzuki, K.; Kiyohara, T. Influence of water stress on development of pine wilting disease caused by Bursaphelenchus lignicolus. Eur. J. For. Pathol. 1978, 8, 97-107. [CrossRef]

16. Kaneko, S. Effect of light intensity on the development of pine wilt disease. Can. J. Bot. 1989, 67, 1861-1864. [CrossRef]

17. Rutherford, T.; Webster, J. Distribution of pine wilt disease with respect to temperature in North America, Japan, and Europe. Can. J. For. Res. 1987, 17, 1050-1059. [CrossRef]

18. Toda, T.; Kurinobu, S. Genetic improvement in pine wilt disease resistance in Pinus thunbergii: The effectiveness of pre-screening with an artificial inoculation at the nursery. J. For. Res. 2001, 6, 197-201. [CrossRef]

19. Toda, T.; Kurinobu, S. Realized genetic gains observed in progeny tolerance of selected red pine (Pinus densiflora) and black pine (P. thunbergii) to pine wilt disease. Silvae. Gent. 2002, 51, 42-44.

20. Hakamata, T. Studies on efficient production technique of Pinus thunbergii seedlings to Bursaphelenchus xylophilus. Tech. Bull. Shizuoka. Res. Ins. Agric. For. 2013, 10, 1-46, (In Japanese with English summary).

21. Kuroda, K.; Ohira, M.; Okamura, M.; Fujisawa, Y. Migration and population growth of the pine wood nematode (Bursaphelenchus xylophilus) related to the symptom development in the seedlings of Japanese black pine (Pinus thunbergii) families selected as resistant to pine wilt. J. Jpn. For. Soc. 2007, 89, 241-248. (In Japanese with English summary) [CrossRef]

22. Aikawa, T.; Kikuchi, T.; Kosaka, H. Demonstration of interbreeding between virulent and avirulent populations of Bursaphelenchus xylophilus (Nematoda: Aphelenchoididae) by PCR-RFLP method. Appl. Entomol. Zool. 2003, 38, 565-569. [CrossRef]

23. Japan Meteorological Agency. 2020 Climate Statistics. Available online: http://www.data.jma.go.jp/obd/stats/ etrn/index.php (accessed on 10 December 2017).

24. R Core Team. R: A Language and Environment for Statistical Computing; R Foundation for Statistical Computing: Vienna, Austria, 2019.

25. Bates, D.; Maechler, M.; Bolker, B.; Walker, S. Fitting linear mixed-effects models using LME4. J. Stat. Softw. 2015, 67, 1-48. [CrossRef]

26. Fox, J.; Weisberg, S. An R Companion to Applied Regression, 3rd ed.; Sage: Thousand Oaks, CA, USA, 2019.

27. Hothorn, T.; Bretz, F.; Westfall, P. Simultaneous inference in general parametric models. Biom. J. 2018, 50, 346-363. [CrossRef] [PubMed]

28. Bartoń, K. MuMIn: Multi-Model Inference; R Package Version 1.43.17; 2020. Available online: https: //CRAN.R-project.org/package=MuMIn (accessed on 1 June 2020).

29. Hirao, T.; Fukatsu, E.; Watanabe, A. Characterization of resistance to pine wood nematode infection in Pinus thunbergii using suppression subtractive hybridization. BMC Plant. Biol. 2012, 12, 13. [CrossRef] [PubMed]

30. Kusumoto, D.; Yonemichi, T.; Inoue, H.; Hirao, T.; Watanabe, A.; Yamada, T. Comparison of histological responses and tissue damage expansion between resistant and susceptible Pine thunbergii infected with pine wood nematode Bursaphelenchus xylophilus. J. For. Res. 2014, 19, 285-294. [CrossRef]

31. Kuroda, K. Inhibiting factors of symptom development in several Japanese red pine (Pinus densiflora) families selected as resistant to pine wilt. J. For. Res. 2004, 9, 217-224. [CrossRef]

32. Nakajima, G.; Iki, T.; Yamanobe, T.; Nakamura, K.; Aikawa, T. Spatial and temporal distribution of Bursaphelenchus xylophilus inoculated in grafts of a resistant clone of Pinus thunbergii. J. For. Res. 2019, 24, 93-99. [CrossRef]

33. Matsunaga, K.; Watanabe, A. Outline of the results of the "Project to advance the development of technology for varieties of Japanese black pine and red pine resistant to the pine wood nematode" commissioned by the Forestry Agency, Ministry of Agriculture, Forestry and Fisheries of Japan-Characterization of pine wood nematode and development of 2nd generation resistant varieties. For. Genet. Breed. 2018, 7, 115-119. (In Japanese)

34. Iwaizumi, M.G. The development of 2nd generation resistant Japanese red pine varieties in collaboration with Prefecture. For. Genet. Breed. 2018, 7, 159-161. (In Japanese)

(C) 2020 by the authors. Licensee MDPI, Basel, Switzerland. This article is an open access article distributed under the terms and conditions of the Creative Commons Attribution (CC BY) license (http://creativecommons.org/licenses/by/4.0/). 